\title{
Desempenho de Sintonizador Online utilizando Lógica Fuzzy para Controle Preditivo Generalizado (GPC) Simuladas em Plantas Industriais Inicialmente Sintonizadas via Algoritmo Genético
}

\author{
Rodrigo Contarato*. Gustavo Maia** \\ *Instituto Federal do Espírito Santo \\ BR (Tel: 27 99991-1280; e-mail: Rodrigo.contarato@hotmail.com). \\ **Instituto Federal do Espírito Santo BR (e-mail: gmaia@ifes.edu.br)
}

\begin{abstract}
The techniques of Model Based Predictive Control (MPC) are more oriented to the industries, because they present greater and greater predictability, but their performance is directly related to their tuning. This study aims to present the results of on-line and off-line parameter tuners to minimize natural system problems using generalized predictive drivers (GPC).
\end{abstract}

There are seven industrial plants that represent the majority of industrial processes to validate the algorithm. Plants are tuned off-line via genetic algorithm seeking a faster response and less error. The occurrences are imputable and recurrent. In order to minimize the disturbances presented, an online synthesizer via fuzzy logic will be used to modulate the parameters at the moment of the disturbances. Present the performance of each plant and show a variation of the parameters at the time of the disturbance.

Resumo: As técnicas de Controle Preditivo Baseado em Modelo (MPC) são cada vez mais aplicados nas indústrias, por apresentarem mais robustez e maior previsibilidade, porém seu desempenho está diretamente ligado à sua sintonia. Este estudo visa apresentar o resultado do desenvolvimento de sintonizadores de parâmetros online e offline para minimização de distúrbios naturais do sistema, utilizando controladores preditivos generalizados (GPC).

São utilizadas sete plantas industriais que representam a maioria dos processos industriais para validar o algoritmo. As setes plantas são sintonizadas de forma off-line via algoritmo genético buscando a resposta mais rápida e com menor erro. Para essas plantas são imputados distúrbios e verificado sua resposta. Com o intuito de minimizar os distúrbios apresentados, será utilizado um sintonizador online via lógica fuzzy para modular os parâmetros no instante dos distúrbios. É apresentando o desempenho de cada planta e demonstrado a variação dos parâmetros no instante do distúrbio.

Keywords: disturbance; online; algorithm; parameter; process.

Palavras-chaves: distúrbio; online; algoritmo; parâmetros; processo.

\section{INTRODUÇÃO}

De acordo com (Aström, K. J, 2001) cerca de 90\% de todos os controladores utilizados na indústria são PID, normalmente é a primeira solução testada em plantas que utilizam feedback. A razão por isso é devido à dificuldade de sintonizar um controlador avançado e equipe qualificada para implementar esses controles. O artigo científico serve para desmistificar esse número e fomentar o assunto para aumentar o número de processos que utilizam controle avançado em indústrias, buscando o melhor controle.

O controle preditivo baseado em modelo (MPC) se refere a uma classe de controladores computacionais que utilizam um modelo explicito do processo para predizer a resposta futura da planta (S. J. Qin and T. A. Badgwell,
2003). Uma variedade de processos pode ser controlada utilizando MPC, desde processos com dinâmica simples até processos com grande atraso ou de fases não mínima, veja (J. Maciejowski, 2002), (J. A. Rossiter 2003) e (P. R. Murath 1998). De acordo com (Camacho, E. F, 2004), no mundo do MPC existem vários algoritmos para modelagem e controle da planta, os métodos mais representativos são DMC, MAC, GPC, PFC, EPSAC e EHAC. O algoritmo MPC normalmente obtém uma performance e robustez muito boa, desde que seus parâmetros estejam bem dimensionados (horizonte de controle $(\mathrm{Nu})$, horizonte de predição $(\mathrm{N} 2)$, fator de supressão $(\lambda)$, ponderação da previsão do erro $(\delta)$ e o fator de previsão de referência $(\alpha)$. As variações desses parâmetros alteram a robustez, tempo de subida e sobressinal. A diminuição do fator de supressão $(\lambda)$, deixa a resposta mais lenta e suave, no aumento proporciona uma resposta mais 
rápida e com maior sobressinal. $\mathrm{O}$ incremento no horizonte de previsão e controle, contribui para melhorar a estabilidade do controlador, o sobressinal e o tempo de subida (G. M. de Almeida, 2016). Existem vários algoritmos para sintonia de controladores MPC, em (J. L. Garriga and M. Soroush, 2010) apresenta um resumo dos algoritmos de sintonia automática, como enxame de partículas (Partical Swarm Optmation PSO) e algoritmo genético (Genetic Algorithm - GA) para controladores MPC do tipo DMC e GPC para uma planta de reator de tanque contínuo agitado, em (J. L. Garriga and M. Soroush, 2010) sintoniza apenas os parâmetros Nu,N2 e $\lambda$, e o critério de escolha da melhor sintonia foi o erro do controle para o setpoint.

Para qualquer sistema de controle, seja ela mais robusta ou mais assertiva, o sistema depende da sintonia do controle, se não estiver bem sintonizado o controle perde desempenho podendo levar a instabilidade.

A diferença deste artigo para os demais artigos já citados será o aumento dos parâmetros de sintonia, no caso $(\mathrm{Nu}, \mathrm{N} 2, \lambda, \delta, \alpha)$ e será apresentado a sintonias deste controlador GPC utilizando (GA - Genetic Algorithm), será levado em conta não somente o erro de predição mas também o tempo de sintonia de cada algoritmo. Os modelos utilizados nesse artigo para sintonia, são 7 funções que representam a maioria dos processos industrias, tais processos possuem dinâmicas diferentes, processos com dinâmicas rápidas, balanceadas, dinâmicas demoradas, alto e baixo tempo morto, de fase mínima e não mínima, tais plantas são consideradas como funções benchmark.

Após a primeira sintonia, sintonia off-line, é empregado a lógica fuzzy para que em tempo real seja feita a avaliação dos distúrbios ou mudanças de setpoint, para então ajustar dinamicamente todos os parâmetros de sintonia, baseado nas regras de controle, objetivando assim, a melhor sintonia possível em tempo real, baseada nas suas regras linguística.

A organização do artigo será da seguinte maneira: seção 2 descreve a formulação do algoritmo GPC irrestrito, na seção 3 descreve o método de sintonia offline (Algoritmo genético (GA) e o método de sintoniza online utilizando lógica fuzzy, na seção IV descreve as 7 funções benchmark para validação das sintonias, na seção $\mathrm{V}$ aborda os resultados obtidos para cada sintonia e na seção VI apresenta a conclusão e trabalhos futuros.

\section{GENERALIZADO (GPC) \\ 2. CONTROLADOR}

O controlador preditivo generalizado foi proposto por (D.W. Clarke, 1987) em 1987 e desde então tem sido utilizado com sucesso em várias aplicações na indústria, pois o controlador pode ser aplicado em sistemas estáveis, instáveis, com elevado tempo morto entre outros. Podemos observar em (ELLIS, Matthew, 2014) uma aplicação recente do MPC em uma planta industrial aplicado em um reator de tanque agitado continuamente. E não só na indústria vem sendo aplicado essa técnica de controle. Pode ser visto em sistemas eletrônicos de potência, como (VAZQUEZ, Sergio,
2017), em sistemas de controle fotovoltaicos (SULTANA, W. Razia, 2017), entre várias outras aplicações.

O modelo utilizado para predição da planta no GPC pode ser o CARMA (Controlled Auto-Regressive and Moving-Average) ou CARIMA (Controlled Auto-Regressive and integrated moving-average). Porém o modelo CARMA considera as perturbações estacionárias, o que é inadequado para o meio industrial. Para trazer o modelo para um meio mais real possível foi criado o modelo CARIMA, que é representado por:

$$
A\left(z^{-1}\right) y(t)=z^{-d} B\left(z^{-1}\right) u(t)+\frac{C\left(z^{-1}\right)}{\Delta} e(t),
$$

onde $\Delta=\left(1-z^{-1}\right)$ de maneira que $\Delta y(t)=y(t)-y(t-1)$,

$$
\begin{aligned}
& A\left(z^{-1}\right)=1+a_{1} \cdot z^{-1}+a_{2} \cdot z^{-2}+\ldots+a_{n a} \cdot z^{-n a} \\
& B\left(z^{-1}\right)=b_{0}+b_{1} \cdot z^{-1}+b_{2} \cdot z^{-2}+\ldots+b_{n b} \cdot z^{-n b} \\
& C\left(z^{-1}\right)=1+c_{1} \cdot z^{-1}+c_{2} \cdot z^{-2}+\ldots+c_{n c} \cdot z^{-n c}
\end{aligned}
$$

A variável em (1) é a saída do sistema; $u(t)$ é o sinal de controle (entrada, ou variável manipulada); ${ }^{e(t)}$ é um ruído branco, que pode representar incertezas de modelagem, erros de medição ou ruídos presentes na saída; d é o atraso de transporte discreto onde $d \mathrm{~T} \leq L \leq(d+1) \mathrm{T}$, T e $\mathrm{L}$ são, respectivamente o período de amostragem e o atraso de transporte contínuo (LJUNG, L. E GLAD, 1994).

O GPC utiliza um modelo interno para gerar as previsões futuras do comportamento do processo, representado pelo modelo CARIMA (1) com o polinômio $\mathrm{C}=1$. O preditor $\mathrm{k}$ passos a frente da saída deste modelo $(\hat{y}(t+k))$, que minimize a variância do erro de previsão que, é obtido através da seguinte expressão:

$$
\hat{y}(t+k)=E[y(t+k) \mid y(0), \ldots, y(t)],
$$

Onde $E[. \mid$.$] é o valor esperado condicional.$ Avançando $\mathrm{k}$ instantes de tempo os termos do modelo CARIMA (1), observamos através de (3) que a saída prevista dependerá das entradas e saídas no instante atual t e nos instantes passados e futuros. No entanto, desejamos que a saída prevista não dependa das saídas futuras, ou seja, das saídas após o instante atual $t$. Para que isto seja possível, utilizaremos no cálculo da previsão, a equação Diophantina (3) definida por:

$$
1=E_{k}\left(z^{-1}\right) A\left(z^{-1}\right)+z^{-k} F_{k}\left(z^{-1}\right),
$$

Onde Ek e Fk são polinômios com graus k-1 e na respectivamente, unicamente definidos através de $\mathrm{k}$ divisões sucessivas de 1 por $\tilde{A}\left(z^{-1}\right)=\triangle A\left(z^{-1}\right)=\left(1-z^{-1}\right) A\left(z^{-1}\right)$. O 
resto e o quociente desta divisão são iguais aos polinômios $z^{-k} F_{k}\left(z^{-1}\right)$ e $E_{k}\left(z^{-1}\right)$, respectivamente.

Avançando $\mathrm{k}$ instantes os termos da equação (1) com $\mathrm{C}=1$ e multiplicando o resultado por $\Delta$ concluímos;

$\tilde{A}\left(z^{-1}\right) y(t+k)=B\left(z^{-1}\right) \Delta u(t+k-d-1)+e(t+k)$

Multiplicando (5) por $E_{k}$ e usando (4), concluímos que

$$
\begin{aligned}
& y(t+k)=E_{k}\left(z^{-1}\right) B\left(z^{-1}\right) \Delta u(t+k-d-1)+\ldots \\
& \ldots+F_{k}\left(z^{-1}\right) y(k)+E_{k}\left(z^{-1}\right) e(t+k)
\end{aligned}
$$

Observe que os termos do polinômio $E_{k}\left(z^{-1}\right) e(t+k)$ estão no futuro, tendo em vista que o polinômio $E_{k}$ tem grau k-1. Logo, aplicando o operador valor esperado em (5) obtemos

$$
\begin{aligned}
& \hat{y}(t+k)=\ldots \\
& \ldots=E_{k}\left(z^{-1}\right) B\left(z^{-1}\right) \Delta u(t+k-d-1)+F_{k}\left(z^{-1}\right) y(k)=\ldots \\
& \ldots=G_{k}\left(z^{-1}\right) \Delta u(t+k-d-1)+F_{k}\left(z^{-1}\right) y(t)
\end{aligned}
$$

Onde

$$
\begin{aligned}
& G_{k}\left(z^{-1}\right)=E_{k}\left(z^{-1}\right) B\left(z^{-1}\right)=\ldots \\
& \ldots=g_{0}+g_{1} z^{-1}+g_{2} z^{-2}+\ldots+g_{n b+j-1} z^{-(n b+k-1)}
\end{aligned}
$$

Note que o polinômio $G_{k}\left(z^{-1}\right) \Delta u(t+k-d-1)$, $k=d+1 \ldots, d+N$, para $N=N_{2}-d$, possui termos com ações de controle passadas, presente e futuras. Para separálos, definimos os polinômios $H_{k}\left(z^{-1}\right)$ e $\bar{H}_{k}\left(z^{-1}\right)$ com ordens k-d-1 e $n_{b}+k-1$, respectivamente, tais que, onde:

$$
H_{k}\left(z^{-1}\right)=g_{0}+g_{1} z^{-1}+\ldots+g_{k-d-1} z^{-(k-d-1)}
$$

Concluímos que $\quad H_{d+l}\left(z^{-1}\right) \Delta u(t+l-1)$, $l=k-d+l, l=1 \ldots, N$, para $N=N_{2}-d$, possui termos com ações de controle presente e futuras, enquanto que $\bar{H}_{d+l}\left(z^{-1}\right) \Delta u(t+l-1)$ possui termos com ações de controle passadas. Através do Lema 1 de (G. M. de Almeida, 2006), chegamos à seguinte expressão da predição do modelo baseado em função de transferência:

$$
\begin{aligned}
& \hat{y}(t+d+l)=H_{d+l} \Delta u(t+l-1)+\ldots \\
& \ldots+z^{l} \bar{H}_{d+l}\left(z^{-1}\right) \Delta u(t-1)+F_{d+l}\left(z^{-1}\right) y(t)
\end{aligned}
$$

A partir destas equações, usamos o Lema 2 de (G. M. de Almeida, 2006) para determinar a expressão matricial:

$$
Y=G \Delta u+\bar{H}\left(z^{-1}\right) \Delta u(t-1)+F\left(z^{-1}\right) y(t)
$$

A resposta livre (depende apenas de ações passadas) pode ser considerada como:

$$
\Phi(t)=\bar{H}\left(z^{-1}\right) \Delta U(t-1)+F\left(z^{-1}\right) y(t)
$$

Portanto, a expressão de previsão na forma matricial segue

$$
Y=G \Delta U+\Phi
$$

A solução do MPC linear é obtida determinando o vetor de sinais de controle $\Delta U$, através da minimização da função custo.

$$
J=\begin{aligned}
& \delta(G \Delta U+\Phi-W)^{T}(G \Delta U+\Phi-W)+\ldots \\
& +\ldots \lambda \Delta U^{T} \Delta U
\end{aligned}
$$

Assim, derivando a função custo $\mathrm{J}$ em relação a $\Delta U$ e igualando o resultado a zero, ou seja:

$$
\begin{aligned}
& \frac{d J}{d \Delta U}=\delta\left[2 \cdot\left(G^{T} \cdot G\right) \cdot \Delta U+2 \cdot G^{T} \cdot(\Phi-W)\right]+\ldots \\
& \ldots+2 . \lambda \cdot \Delta U=0
\end{aligned}
$$

Determinamos, através da expressão acima, o vetor de ações de controle presente e futuros:

$$
\Delta U=\left(\delta \cdot\left(G^{T} . G\right)+\lambda . I\right)^{-1} \cdot \delta \cdot G^{T} \cdot(W-\Phi)
$$

O sinal de controle aplicado na planta no instante $t$ é igual a $u(t)=u(t-1)+\Delta u(t)$, onde $\Delta u(t)=K \cdot(W-\Phi)$, e K é a primeira linha da matriz $\delta\left(G^{T} \cdot G+\lambda . I\right)^{-1} \cdot \delta \cdot G^{T}$.

O algoritmo GPC possui os seguintes passos:

- Passo 1) Dados os parâmetros de sintonia e o modelo da planta, determine a matriz de coeficientes $\mathrm{G}$;

- Passo 2) Em cada período de amostragem t, determine o vetor de referência $W(t)$ e o vetor da resposta livre $\Phi(t)$;

- Passo 3) Determine a variação do sinal de controle atual $\Delta u(t)$ através de (18) e o sinal de controle atual. 
- $\quad$ Passo 4) Aplicar o sinal $u(t)$ na planta, fazer $t=t+1$ e retornar ao passo 2 .

\section{MÉTODOS DE SINTONIA ONLINE E OFFLINE}

Os métodos de sintonia são usados para determinar os parâmetros que melhorem o desempenho/robustez de um sistema de controle. No caso dos métodos on-line, os critérios são testados e o parâmetros corrigidos a cada intervalo de tempo.

A metodologia aqui apresentada corrige os parâmetros do MPC de forma a conduzir o processo para uma especificação projetada no domínio do tempo, denominada de túnel de desempenho. Esses túneis são projetados tanto para o problema servo, como para o problema regulatório, e podem ser usados para redução de overshoot, correção de tempo de resposta, entre outros critérios (Ali and Al-Ghazzawi, 2008). As correções nos parâmetros são feitas através de um FIS (Fuzzy Inference System), que tem como referência a predição do sistema em malha fechada.

A aplicação deste algoritmo de sintonia online é dividida em duas etapas. A primeira consiste no monitoramento da predição do sistema em malha fechada, e no caso de violação dos limites especificados ou na mudança de set-point, o algoritmo entra na segunda etapa. Na segunda etapa, os túneis de desempenho são escalonados proporcionalmente à magnitude do distúrbio ou mudança de set-point e então as predições são usadas no FIS para determinar os novos parâmetros (Al-Ghazzawi et al., 2001).

Nesta etapa, uma variável medida específica é transformada em um membro de conjuntos difusos. Dois conjuntos fuzzy de entrada e um conjunto fuzzy de saída serão usados neste trabalho. O primeiro conjunto de entrada é mostrado na Figura 1.

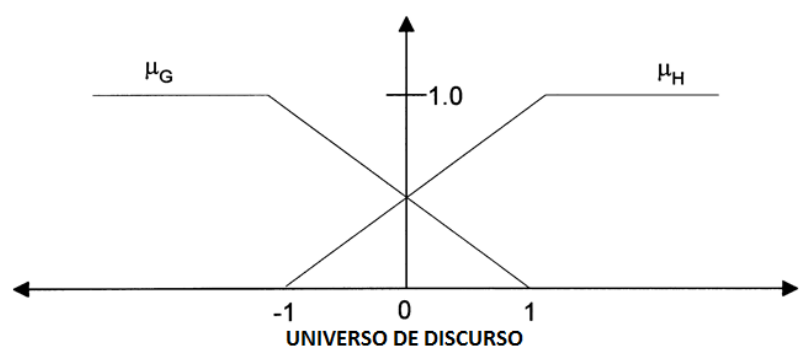

Figura 1 - Conjunto de entrada fuzzy A e B.

O conjunto consiste em duas funções de pertinências trapezoidal, a saber; Bom denotado como $\mu \mathrm{g}$ e Alto denotado como $\mu \mathrm{h}$. O universo do discurso deste conjunto abrange os valores possíveis para sua entrada específica, que é o valor escalado para a violação vinculada. A violação é definida da seguinte forma:

Se o limite superior for violado:

$$
A \equiv \frac{y_{j}(k+m)-y_{j}^{u}(k+m)}{y_{j}^{u}(k+m)}
$$

Se o limite inferior for violado:

$$
B \equiv \frac{y_{j}^{L}(k+m)-y_{j}(k+m)}{y_{j}^{L}(k+m)}
$$

onde: $y_{j}$ é o valor previsto da $j^{\text {th }}$ saída, $y^{\mathrm{u}}$ e $y^{1}$ são os limites máximos e mínimos de y. Além disso, $\mathrm{k}$ é o tempo de amostragem e m é o instante no qual a violação máxima ocorre para a $\mathrm{j}^{\text {th }}$ saída. A definição de A e B nas equações (19) e (20) garante valor positivo se o limite correspondente for violado e negativo caso contrário. Observe que os limites superior e inferior não podem ser violados no mesmo tempo por uma saída específica. Portanto, se o limite superior for violado, então A pertence a $\mu H$ e B para $\mu G$ e vice-versa. Se nenhum limite for violado, então A e B pertence a $\mu G$. Este argumento se aplica para o caso de mudança de set point. Também se aplica ao caso de perturbação, com a exceção de que o pertencimento de $B$ às funções de associação que representam os conjuntos difusos é invertido. A razão para reverter é que o limite inferior no caso de perturbação é sempre negativo porque lidamos com variáveis de desvio neste trabalho.

O segundo conjunto fuzzy de entrada é mostrado na Figura 2.

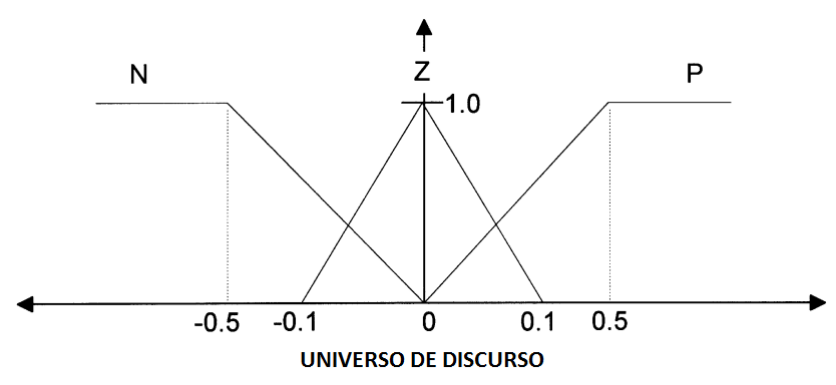

Figura 2 - Conjunto de entrada fuzzy C.

$\mathrm{O}$ conjunto consiste em três funções, a saber; (P)ositivo, (Z)ero e (N)egativo. Estas funções são designadas $\mu \mathrm{P}, \mu \mathrm{Z}$ e $\mu \mathrm{N}$, respectivamente. A entrada para este conjunto difuso é a taxa de violação escalada, que é definida da seguinte forma:

$$
C \equiv \frac{y_{j}(k+m)-y_{j}(k+m-1)}{y_{j}(k+m)}
$$

A equação (21) para taxa de violação se aplica à violação de limite superior e inferior. Observe que os valores em escala são usados para a medida de violação vinculada (A, B) e sua taxa (C). A razão para isso é simplificar a determinação do intervalo possível para o universo do discurso nas Figuras xxx.

As regras básicas que governam as diretrizes de ajuste representam a saída da regra para $\lambda, \gamma$ e $\mathrm{P}$, respectivamente. Os acrônimos LN, SN, ZE, SP e LP são os conjuntos difusos de saída para $\lambda$, $\gamma$ e P, como mostrado na figura 3 . 


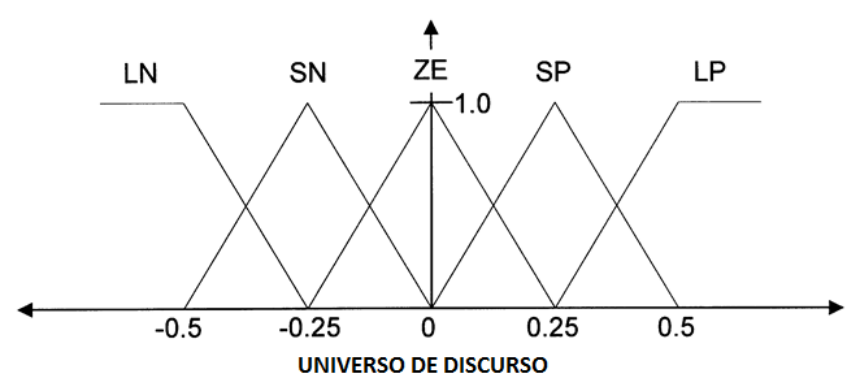

Figura 3 - Conjunto de saída fuzzy.

Sabe-se que o aumento de $\lambda$ torna a resposta mais lenta e estável. Em valores próximos de zero, $\lambda$ torna a resposta mais agressiva. A resposta pode ser oscilatória ou subamortecida dependendo da dinâmica do processo e do valor de h_p. No caso $\lambda$ é ligeiramente aumentado de zero, enquanto outros parâmetros são fixos, a resposta fica mais lenta e a resposta pode exibir apenas um comportamento abaixo do amortecido se não fosse o caso quando $\lambda$ tende a zero. Quando $\lambda$ é aumentado para além de um valor crítico, o comportamento sob amortecimento desaparece e a resposta torna-se muito lenta. $O$ valor crítico para $\lambda$ aumenta com o aumento de $h \_p$. Com base no entendimento acima, é sempre preferível reduzir $\lambda$ para acelerar a resposta, ou seja, quando o limite inferior das especificações de desempenho é violado. Da mesma forma, é desejável reduzir $\lambda$ a fim de eliminar o overshoot, ou seja, quando o limite superior é violado. Geralmente, $\lambda$ é aumentado apenas quando a redução não produz nenhum efeito adicional. O raciocínio acima sobre $\lambda$ é refletido nas regras básicas do algoritmo.

Na defuzzificação, os resultados do mecanismo de inferência são combinados para produzir um valor nítido para a saída. A saída obtida é o fator que será usado para ajustar os parâmetros de ajuste do MPC. O procedimento de defuzzificação é basicamente semelhante a média ponderada. Existem várias maneiras de realizar o procedimento de defuzzificação (Yen e Langari, 1999). Aqui será adotado o método do centro de área (COA).

O algoritmo genético é um método evolutivo de otimização introduzido por (Holland, J. H., 1975) em 1975. O GA se inspira nos princípios da teoria da seleção natural, proposta por Charles Darwin, na qual somente os indivíduos mais aptos sobrevivem para uma próxima geração. O GA utiliza uma população de indivíduos (chamada também de cromossomos), essa população será submetida a 3 operadores: seleção, crossover (cruzamento) e mutação. Aplicando estes operadores de maneira iterativa, ao fim de um critério de parada pré-estabelecido, o algoritmo entrega a otimização desejada (ou algo próximo dela). Em (Ellis, Durand, 2014) é utilizado essa sintonia por GA e comparada com os métodos clássicos de sintonia não automática, nessa comparativa o GA obteve melhor resultado em todos os parâmetros, robustez, velocidade de subida, menor erro mínimo (ISE).

O processo proposto por (Holland, J. H., 1975) tem o seguinte roteiro:
- $\quad 1^{\circ}$ Criação de uma população aleatória

- $\quad 2^{\circ}$ Seleção dos melhores indivíduos da população, ou seja, que possua menor valor na função objetivo (caso o retorno da função objetivo seja o erro).

- $\quad 3^{\circ}$ Crossover (cruzamento), nesta etapa é realizado o cruzamento entre indivíduos com intuito de gerar novos indivíduos. Esse cruzamento é feito por meio de uma combinação linear entre dois indivíduos.

- $\quad 4^{\circ}$ Mutação, este operador também auxilia na diversidade e nada mais é do que a inserção de um ruído alguns indivíduos da população.

$5^{\circ}$ Critério de parada, após o $4^{\circ}$ passo é verificado se o critério de parada foi atingido, caso não, volta para o $2^{\circ}$ passo.

Para o funcionamento do algoritmo é necessário definir alguns parâmetros como, tamanho da população, quantidade de variáveis, taxa de mutação, taxa de cruzamento, número máximo de interações e a função objetivo.

\section{FUNÇÕES BENCHMARKTING PARA VALIDAÇÃO DA SINTONIA}

Para comparar os algoritmos de sintonia automática do GPC, foi simulado quatro classes de modelos que, segundo (ÅSTRÖM, Karl Johan; HÄGGLUND, Tore, 1995) e (Segovia, V. Romero ; Hägglund, T. ; Åström, K.J., 2014), podem representar a maioria dos processos comumente encontrados na indústria, os quais são descritos pelas seguintes funções de transferências:

$$
\begin{gathered}
G_{1 ; \mu}=\frac{1}{(\mu s+1)^{2}} \text { para } \mu=0,1 ; 3 ; 10 ; \\
G_{2}=\frac{1}{(s+1)^{4}} ; \\
G_{3 ; \mu}=\frac{1}{(s+1)(s+\mu)\left(s+\mu^{2}\right)\left(s+\mu^{3}\right)} \text { para } \mu=0,2 ; 0,7 ; \\
G_{4}=\frac{1-0,1 s}{(s+1)^{3}} ;
\end{gathered}
$$

Considerando os diferentes parâmetros de $\mu$ para cada classe de função de transferência, teremos ao todo, sete modelos. Cada função de transferência tem uma característica importante, como, resposta rápida, resposta moderada, resposta lenta, alto tempo morto, baixo tempo morto, fase não mínimo e fase mínima.

Para a aplicação do GPC, foi preciso discretizar a função de transferência continua. $O$ processo de discretização foi realizado da seguinte forma, para cada função de transferência foi aplicado um degrau unitário e foi obtido a resposta do sistema, desta forma, foi observado o tempo que 
se leva para alcançar os $63,2 \%$ da resposta estabilizada (constante de tempo) e de acordo com a taxa de amostragem de Nyquist foi definido um tempo de amostragem de 10 vezes menor que a constante de tempo. Dessa forma, foram discretizados as 7 funções de (22).

\section{RESULTADOS DAS SINTONIAS}

Para obter a melhor análise das sintonias foram observados dois fatores em cada simulação, o primeiro é o erro de cada sintonia e o segundo fator é o tempo de resposta. Para obter o erro foi calculado o MSE (Erro Quadrático Médio) do valor predito para o valor desejado e para obter o tempo de processamento de cada simulação foi utilizado a função Tic Toc do próprio Matlab.

O programa utilizado para as simulações foi o Matlab 2016b e foi utilizado um notebook com processador core i5-4200U CPU $1.60 \mathrm{GHz}$, com 8GB de memória RAM no sistema operacional Windows 8.1 .

Foram escolhidas as variáveis $(\lambda, \delta, \alpha, N 2, N u)$ para sintonizar a planta, pois elas representam toda a dinâmica da resposta, tempo de subida, sobressinal, entre outros. O objetivo dos algoritmos é achar uma combinação entre as variáveis que tenha o menor erro médio quadrático (MSE), ou seja, vai buscar os resultados que tenham as respostas mais rápidas, com menor sobressinal (sobressinal aumenta o erro) e com menor oscilação. O erro quadrático médio é comparado com a referência (setpoint), quanto mais rápido e menos oscilatório for a resposta menor será o MSE.

A tabela 1 apresentam os parâmetros achados pela sintonia utilizando os algoritmos (GA), apresenta também as duas funções de desempenho estudadas, o MSE e o tempo de processamento.

Tabela 1 - Desempenho da sintonia do GA

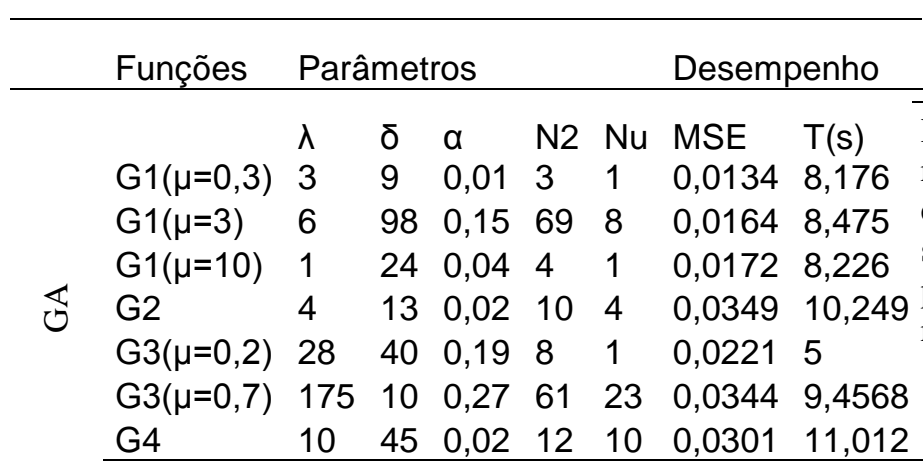

As sintonias off-line buscam a melhor resposta para a dinâmica simulada. Para testar a sintonia, foi implantado distúrbios nas plantas. Com o distúrbio, as respostas das plantas são alteradas e os parâmetros inicialmente sintonizados já não tem uma boa resposta. A figura 4 representa o distúrbio aplicado nas plantas. As plantas seguem a referência de saída com amplitude igual a 1 e o distúrbio aplicado foi um degrau de amplitude 2 no instante de 50 a 80 amostras e outro distúrbio de amplitude 3 no instante 100 a 150 amostras.

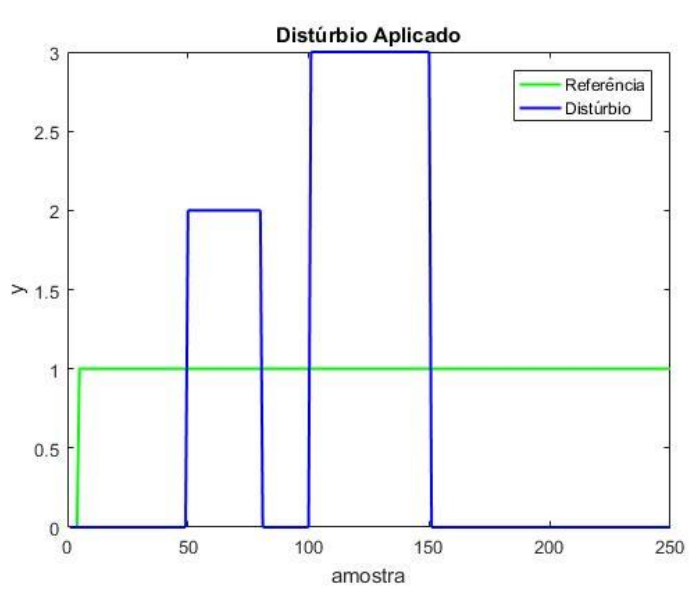

Figura 4 - Distúrbio aplicado na planta.

Para ambas as simulações os controladores foram sintonizados de forma off-line, porém uma simulação utiliza sintonia online. A tabela 2 abaixo apresenta os resultados obtidos. Para as plantas sintonizadas apenas de forma off-line é utilizado a sigla (AG - Algoritmo Genético) e para as plantas sintonizadas de forma online recebe o nome de (AGFO - Algoritmo Genético Fuzzy Online).

Tabela 2 - Desempenho da sintonia online utilizando lógica fuzzy.

\begin{tabular}{llll}
\hline \multicolumn{3}{c}{ Desempenho } \\
\hline & Funções & MSE \\
\hline & & AG & AG-FO \\
1. & G1 $(\mu=0,3)$ & 1,2930 & 1,1889 \\
2. & G1 $(\mu=3)$ & 2,2693 & 1,8756 \\
3. & G1 $(\mu=10)$ & 1,8608 & 1,4747 \\
4. & G2 & 52,922 & 42,544 \\
5. & G3 $(\mu=0,2)$ & 2,9413 & 1,9699 \\
6. & G3 $(\mu=0,7)$ & 47,433 & 30,753 \\
7. & G4 & 19,332 & 15,667 \\
\hline
\end{tabular}

Para exemplificação destes resultados, será apresentado a resposta de duas sintonias, a sintonia da planta G3 para $\mu=0,7$ e a planta G4. Neste resultado é apresentado a comparação da sintonia off-line e a sintonia online apresentando os parâmetros alterados. Segue a figura 5 apresentando a resposta da sintonia $\mathrm{G} 3$ para $\mu=0,7$.
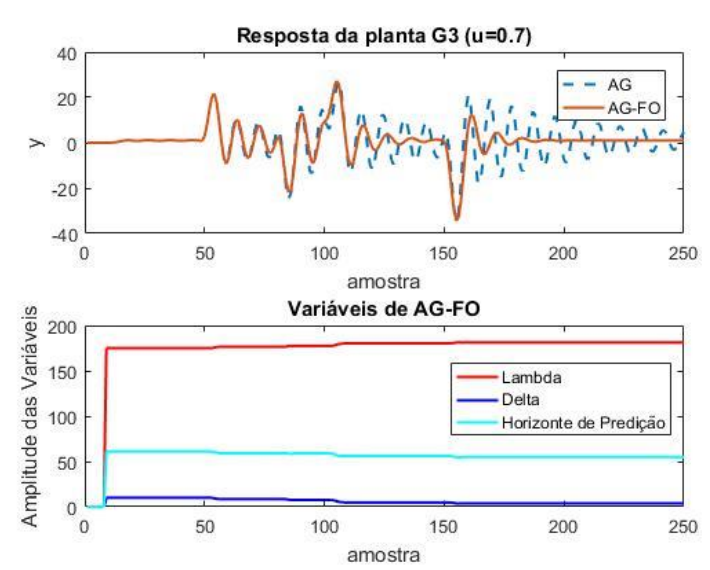
Figura 5 - Resposta da planta G3 para $\mu=0,7$.

Nesta planta, a sintonia off-line ainda é muito oscilatória e apresenta alta amplitude na presença do distúrbio, mesmo com sintonia de lambda iniciando em 175, o algoritmo acresce o lambda e diminui o delta para tornar a resposta menos oscilatória.

Na figura 6 é apresentado a sintonia da planta G4.
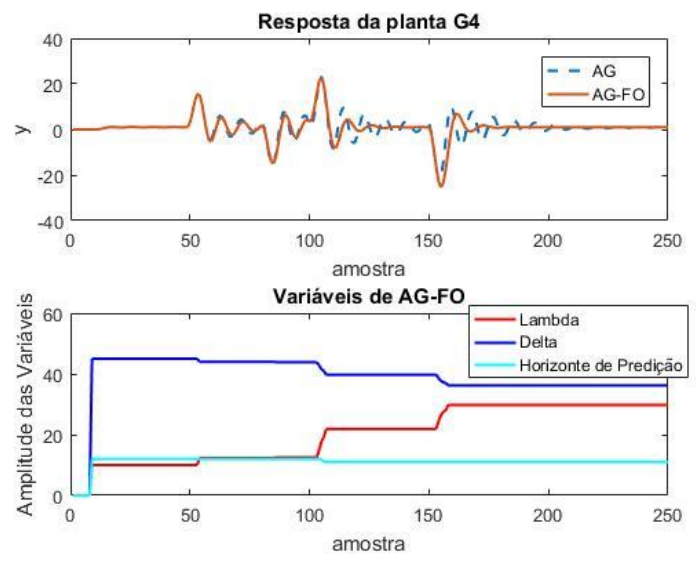

Figura 6 - Resposta da planta G4.

Para esta planta que tem uma dinâmica rápida, está com uma sintonia menos robusta, o algoritmo faz uma logística parecida ao apresentado na planta anterior e busca tornar a resposta menos oscilatória, aumentando o lambda e diminuindo o delta, está alteração faz com o que a resposta seja menos oscilatória tornando-o mais robusto.

\section{CONCLUSÃO}

Analisando os dados apresentados para a simulação, pode ser observado que o tempo de sintonia para é pequeno, e os erros obtidos são bastante satisfatórios.

Para qualquer sistema de controle, seja ela mais robusta ou mais assertiva, o sistema depende da sintonia do controle, se não estiver bem sintonizado o controle perde desempenho podendo levar a instabilidade. Conforme visto na secção 5, as plantas sintonizadas via algoritmo genético, algumas delas foram sintonizadas de forma a ter uma resposta rápida, com isso quando é aplicado distúrbio, as plantas apresentam grande variação.

A contribuição deste artigo está na correção dos parâmetros de forma online utilizando lógica fuzzy, quando o sistema está sobre a presença de distúrbios ou mudança de setpoint. Para as plantas que representam a maioria dos processos industriais, descrito na secção 4 , foi observado que a sintonia online teve resultados ótimos, reduzindo o erro médio em no mínimo $12 \%$, o sobressinal e tornando o controle mais robusto.

\section{REFERÊNCIAS}

Ali, Emadadeen \& Al-Ghazzawi, Ashraf. (2008). On-line Tuning of Model Predictive Controllers Using Fuzzy
Logic. The Canadian Journal of Chemical Engineering. 81. 1041 - 1051 .

Al-Ghazzawi, A., Ali, E., Nouh, A. and Zafiriou, E. (2001). On-line tuning strategy for model predictive controllers, Journal of Process Control 11(3): 265-284

Aström, K. J.; Hägglund,T. "The future of pid control". Control Engineering Practice, 9,pp 1163-1175, 2001.

Astrom, Karl Johan; HÄGGLUND, Tore. PID controllers: theory, design, and tuning. Research Triangle Park, NC: Instrument society of America, 1995.

Camacho, E. F., and Bordons, C. Model Predictive Control. New York: Springer,2nd edition, 2004.

D.W. Clarke, C. Mohtadi, and P.S. Tuffs. Generalized Predictive Control. Part I. The Basic Algorithm. Automatica, 23(2):137-148, 1987.

Ellis, Matthew; Durand, Helen; Christofides, Panagiotis D. A tutorial review of economic model predictive control methods. Journal of Process Control, 2014, 24.8: 11561178.

G. M. de Almeida and J.L.F. Salles Controle preditivo: sintonia e aplicações na siderurgia, 1rd ed, Appris, 2016.

G. M. de Almeida, Sintonia de controle preditivo generalizado através de algoritmo genético. , Dissertação (Mestrado), Programa de Pós Graduação em Engenharia Elétrica, PPGEE/UFES, Vitória/ES, 2006.

Holland , J. H. (1975). Adaptation in Natural an d Artificial Systems. MIT Press.

J. A. Rossiter Model-Based Predicitve Control: A Pratical Approach, Boca Raton, FL: CRC Press, 2003.

J. L. Garriga and M. Soroush Model Predictive Control Tuning Methods: A Review) Preocesses, Ind. Eng. Chem. Res., 2010.

J. Maciejowski Predictive Control with Constraints , Englewood Cliffs, NJ: Prentice Hall, 2002.

Ljung, L. E Glad, T. Modeling of Dynamic Systems, Prentice-Hall, Englewood Cliffs, NJ, 1994

P. R. Murath and D. A. Mellichamp and D. E. Seborg Predictive Controller Design for Single Input/Single Output System, Ind. Eng. Chem. Res., vol 27, 1998

Segovia, V. Romero ; Hägglund, T. ; Åström, K.J. Measurement noise filtering for PID controllers. Journal of Process Control, April 2014, Vol.24(4), pp.299-313.

S. J. Qin and T. A. Badgwell A Survey Of Industrial Model Predictive Control Technology Control Engineering Practices, v11, 2003.

Sultana, W. Razia, et al. A review on state of art development of model predictive control for renewable energy applications. Renewable and Sustainable Energy Reviews, 2017, 76: 391-406.

Vazquez, Sergio, et al. Model predictive control for power converters and drives: Advances and trends. IEEE Transactions on Industrial Electronics, 2017, 64.2: 935947.

Yen e Langari, 1999 Fuzzy Logic: Intelligence, Control, and Information, 548 pages, 